\title{
Effects of a Phase III Cardiac Rehabilitation Program on Physical Status and Lipid Profiles in Elderly Patients With Coronary Artery Disease Juntendo Cardiac Rehabilitation Program (J-CARP)
}

\author{
Eriko Seki, MD*; Yoshiro Watanabe, MD*; Kazunori Shimada, MD*,**; Satoshi Sunayama, MD*; \\ Tomo Onishi, MS**; Kazunobu Kawakami, BS**; Mizue Sato, RN**; Hiroyuki Sato, MD**; \\ Hiroshi Mokuno, MD*; Hiroyuki Daida, MD*
}

\begin{abstract}
Background Limited data are available regarding the effects of phase III cardiac rehabilitation on the physical status and risk factors in elderly patients with coronary artery disease (CAD).

Methods and Results Thirty-four male CAD patients ( $>65$ years old) were randomly assigned to an intervention group $(n=18)$ or a control group $(n=16)$. The intervention group participated in a phase III cardiac rehabilitation program consisting of exercise training, diet therapy, and weekly counseling for 6 months. In the control group, usual outpatient care was provided. In the intervention group, body mass index, waist size and fat weight significantly decreased; peak $\dot{\mathrm{V}}_{2}$ and anaerobic threshold $\dot{\mathrm{V}}_{2}$ were maintained; isokinetic peak torques of knee extensor and flexor muscles significantly increased; anterior trunk flexibility was significantly improved. In the control group, all parameters were unchanged except for peak $\dot{\mathrm{V}} \mathrm{O}_{2}$, which significantly decreased. In the intervention group, serum total cholesterol levels significantly decreased after cardiac rehabilitation. However, highdensity lipoprotein-cholesterol and apoA-I levels also decreased. In the control group, no significant change in lipid profile was observed.

Conclusions The results suggest that phase III cardiac rehabilitation could be beneficial for elderly patients with CAD. (Circ J 2008; 72: 1230-1234)
\end{abstract}

Key Words: Coronary artery disease; Elderly patients; Exercise; Lipid profile; Phase III cardiac rehabilitation

$\mathbf{T}$

he rapid growth of the elderly population in Japan has led to a remarkable increase in patients with coronary artery disease (CAD). The elderly have a 2- to 3-fold higher incidence of acute myocardial infarction when compared with younger persons. They also have more complications, resulting in prolonged hospital stays with low physical function and substantially higher fatality rates after CAD events! $!^{-3}$ Because of this high rate of mortality and morbidity, primary and secondary prevention are important strategies not only to improve survival but also to maintain the active social life of the elderly population.

Cardiac rehabilitation has been reported as effective for improving coronary risk factors and increasing exercise tolerance in patients with CAD after cardiac events! ${ }^{1-8}$ Although the benefits of cardiac rehabilitation have been reported in elderly as well as younger patients, ${ }^{2-4,7}$ recent reports suggest that elderly patients are not referred to or vigorously encouraged to attend these programs?-11 In

(Received October 29, 2007; revised manuscript received March 3, 2008; accepted April 3, 2008)

*Department of Cardiovascular Medicine, Juntendo University School of Medicine, **Juntendo Sports Clinic, Juntendo University Hospital, Tokyo, Japan

Mailing address: Hiroyuki Daida, MD, Department of Cardiovascular Medicine, Juntendo University School of Medicine, 2-1-1 Hongo, Bunkyo-ku, Tokyo 113-8421, Japan. E-mail: daida@med.juntendo. ac.jp

All rights are reserved to the Japanese Circulation Society. For permissions, please e-mail: cj@j-circ.or.jp general, cardiac rehabilitation programs are performed in 3 stages: acute stage (phase I), subacute stage (phase II), and chronic stage (phase III). In Japan, most cardiac rehabilitation programs have been phase I and some phase II,2,13 whereas phase III cardiac rehabilitation has not often been performed because until March 2006, it was not covered by Japanese health insurance. Therefore, limited data are available regarding the effects of phase III cardiac rehabilitation on psychological and physical status. We previously reported that a phase III cardiac rehabilitation program, namely the Juntendo Cardiac Rehabilitation Program (J-CARP), significantly improved several aspects of the quality of life for elderly patients with CAD!11

In this prospective randomized clinical trial, we assessed the beneficial effects of 6 months of phase III comprehensive cardiac rehabilitation on physical status and coronary risk factors in elderly male Japanese patients with CAD.

\section{Methods}

\section{Subjects}

We prospectively enrolled 39 consecutive male patients who were more than 65 years old with stable CAD attending the outpatient clinic at Juntendo University Hospital. Patients with ongoing congestive heart failure, liver dysfunction (aspartate aminotransferase $\geq 40 \mathrm{IU} / \mathrm{L}$ ), renal dysfunction (creatinine $\geq 2.0 \mathrm{mg} / \mathrm{dl}$ ), or systemic diseases, including malignancy and collagen disease, were excluded. All patients had been referred at least 6 months after a 
major coronary event, such as acute myocardial infarction, coronary artery bypass grafting, or percutaneous coronary intervention. We randomly assigned these patients to an intervention group $(n=20)$ or a control group $(n=19)$. We measured exercise tolerance, muscle strength, and risk factor profiles before and after cardiac rehabilitation. Finally, we obtained data from 34 patients $(n=18$ and $n=16$, respectively) for the present study because we could not measure the blood chemistry for 5 subjects under fasting conditions. In the intervention group, subjects participated in an outpatient phase III cardiac rehabilitation program, while the control group received usual outpatient care, including monthly medical examination by the physician in charge. Except for the rehabilitation protocol, standard medical care was provided for both groups. Lipid-lowering drugs and other medications that may affect lipid levels were given at stable doses for at least 4 weeks before entry, and the doses of these medications were not altered during the study period. Subjects received full verbal and written explanations of the nature and purpose of the study, and each gave written informed consent. The study was approved by the Ethical Committee of Juntendo University.

\section{Rehabilitation Protocol}

In the intervention group, subjects participated in a weekly outpatient phase III cardiac rehabilitation program that included an exercise session, exercise prescription, dietary instruction and an educational program for 6 months as described previously!1 Supervised exercise sessions at the clinic consisted of approximately $15 \mathrm{~min}$ of warm-up exercises including stretching, followed by $20-60 \mathrm{~min}$ of continuous upright aerobic exercise (various combinations of walking, bicycling, jogging, and other activities) and light isotonic exercise such as sit-ups and squatting using the patient's own body weight, followed by approximately $15 \mathrm{~min}$ of cool-down stretching and calisthenics. The intensity of exercise was prescribed individually at the anaerobic threshold (AT) level as measured by a treadmill exercise test using expiratory gas analysis or a rating of $12-13$ on the standard Borg perceived exertion scale. In addition to the weekly supervised exercise sessions, subjects were encouraged to perform aerobic exercise twice weekly ( $\geq 30 \mathrm{~min}$ ) at home at an intensity of heat rate of AT or a rating of 12-13 on the Borg scale. Each patient's exercise prescription was periodically adjusted for a gradual increase on the basis of repeated treadmill testing. In the intervention group, patients were instructed about the phase II diet of the American Heart Association at the beginning and every 2 months of the study. An educational program was also given to each subject by physicians and nurses regarding ischemic heart disease and risk factors at baseline. In addition, individual counseling for physical and psychological conditions was basically provided every visit. Subjects were frequently encouraged by physicians, dietitians, nurses, and exercise physiologists to comply with both exercise and dietary instructions throughout the program.

\section{Evaluation}

In both groups, we evaluated the daily physical activity, physical status, exercise tolerance, strength of thigh muscles, serum lipid levels and glucose tolerance at baseline and after 6 months.

Daily Physical Activity Daily physical activity was evaluated using a pedometer (Lifecorder ${ }^{\circledR}$, Suzuken Co, Ltd, Nagoya, Japan), which can record the mean step count
Table 1 Baseline Characteristics of the Patients

\begin{tabular}{lccc}
\hline \hline & $\begin{array}{c}\text { Intervention } \\
\text { group } \\
(n=18)\end{array}$ & $\begin{array}{c}\text { Control } \\
\text { group } \\
(n=16)\end{array}$ & p value \\
\hline Age (years) & $69 \pm 3$ & $70 \pm 4$ & $N S$ \\
BMI $\left(\mathrm{kg} / \mathrm{m}^{2}\right)$ & $23.4 \pm 2.6$ & $23.8 \pm 3.2$ & $N S$ \\
Hypertension $(\%)$ & $10(56)$ & $5(36)$ & $N S$ \\
Current smoker $(\%)$ & $1(6)$ & $2(14)$ & $N S$ \\
Diabetes mellitus $(\%)$ & $7(39)$ & $5(36)$ & $N S$ \\
Dyslipidemia $(\%)$ & $11(61)$ & $7(44)$ & $N S$ \\
Myocardial infarction $(\%)$ & $9(50)$ & $9(64)$ & $N S$ \\
PCI $(\%)$ & $5(28)$ & $7(50)$ & $N S$ \\
CABG $(\%)$ & $10(56)$ & $4(29)$ & $N S$ \\
Ejection fraction $(\%)$ & $64 \pm 12$ & $66 \pm 15$ & $N S$ \\
Time from last CVE (days) & $1,934 \pm 1,742$ & $1,514 \pm 1,385$ & $N S$ \\
\hline
\end{tabular}

Values are mean value $\pm S D$ or number and percentage in parentheses. $B M I$, body mass index; PCI, percutaneous coronary intervention; $C A B G$, coronary artery bypass grafting; CVE, cardiovascular event (including acute myocardial infarction, $P C I, C A B G)$.

and calculated mean energy of physical activity for up to 42 days.

Physical Parameters Physical parameters were assessed by body mass index (BMI) and waist circumference. The percentages of body fat and lean body weight were measured by the BOD POD ${ }^{\circledR}$ Body Composition System (Life Measurement, Inc, Concord, CA, USA), which analyzes the relationship between pressure and volume to derive the body volume of a subject seated inside a fiberglass chamber. Derivation of body volume, together with measurement of body mass, permits calculation of body density and subsequent estimation of percent fat and fat-free mass.

Exercise Tolerance To assess peak oxygen consumption (peak $\dot{\mathrm{V}} \mathrm{O}_{2}$ ) and the AT, all patients underwent treadmill exercise testing (Marquette Max-1, GE Healthcare BioSciences Corp, Piscataway, NJ, USA) using an expiratory gas analysis machine (Vmax-29S, SensorMedics Co, Yorba Linda, CA, USA). After resting, a warm-up was performed for a few minutes at $2.0 \mathrm{~km} / \mathrm{h}$, followed by ramp loading $(0.6 \mathrm{~km} / \mathrm{h}$ every minute) until subjective exhaustion, progressive angina, ST-segment depression $(\geq 2 \mathrm{~mm})$ or sustained tachyarrhythmia defined by the published guideline ${ }^{14}$ The AT was determined by the "V-slope" method.

Strength of Thigh Muscles Strength of the thigh muscles was measured using the Cybex770 system (Cybex Division of Lumex, Ronkonkoma, NY, USA). The isokinetic peak torque of the knee extensor and of the flexor muscles was measured at $180 \%$ s.

Serum Lipid Profiles Serum lipid profiles were analyzed after at least $12 \mathrm{~h}$ of fasting, and the concentrations of total cholesterol (TC), triglyceride (TG), and high-density lipoprotein-cholesterol (HDL-C) were determined by standard enzymatic methods using an auto-analyzer. Concentrations of low-density lipoprotein-cholesterol were calculated with Friedewald's equation using the concentrations of TC, HDL-C and TG.

\section{Statistical Analysis}

The results are expressed as the mean value \pm standard deviation. Baseline characteristics of the intervention group and the control group were compared using unpaired t-tests. Data at baseline and after 6 months were compared in each patient by paired t-test to evaluate singular effects of cardiac rehabilitation. A p-value of less than 0.05 was considered significant. 
Table 2 Effects of Cardiac Rehabilitation and Exercise Training on Obesity Indices, Exercise Tolerance, Flexibility, and Daily Physical Activity

\begin{tabular}{|c|c|c|c|c|}
\hline & \multicolumn{2}{|c|}{ Intervention group } & \multicolumn{2}{|c|}{ Control group } \\
\hline & At baseline & After 6 months & At baseline & After 6 months \\
\hline \multicolumn{5}{|l|}{ Obesity indices } \\
\hline$B M I\left(k g / m^{2}\right)$ & $23.4 \pm 2.6$ & $22.9 \pm 2.5 * *$ & $23.5 \pm 3.1$ & $23.7 \pm 3.2$ \\
\hline Waist $(\mathrm{cm})$ & $86.1 \pm 7.3$ & $83.9 \pm 7.3 * *$ & $86.9 \pm 9.7$ & $87.3 \pm 9.6$ \\
\hline$\%$ body fat & $25.9 \pm 5.0$ & $25.0 \pm 4.4$ & $27.1 \pm 6.1$ & $26.3 \pm 5.5$ \\
\hline Fat weight $(\mathrm{kg})$ & $16.2 \pm 4.3$ & $15.2 \pm 3.6^{*}$ & $17.5 \pm 6.2$ & $17.0 \pm 5.8$ \\
\hline Lean body weight $(\mathrm{kg})$ & $45.8 \pm 4.8$ & $45.5 \pm 5.1$ & $45.4 \pm 4.8$ & $46.2 \pm 5.7$ \\
\hline \multicolumn{5}{|l|}{ Exercise tolerance } \\
\hline Peak $\dot{\mathrm{V}} \mathrm{O}_{2}\left(\mathrm{ml} \cdot \mathrm{kg}^{-1} \cdot \mathrm{min}^{-1}\right)$ & $22.8 \pm 3.4$ & $24.6 \pm 3.1$ & $24.9 \pm 3.7$ & $22.4 \pm 3.2 * *$ \\
\hline$A T \dot{V} O_{2}\left(\mathrm{ml} \cdot \mathrm{kg}^{-1} \cdot \mathrm{min}^{-1}\right)$ & $12.4 \pm 1.6$ & $13.2 \pm 2.0$ & $12.6 \pm 1.8$ & $12.0 \pm 2.0$ \\
\hline \multicolumn{5}{|c|}{ Strength of thigh muscles isokinetic strength test $\left(180^{\circ} / \mathrm{s}\right)$} \\
\hline Extension, \%Bw & $104.2 \pm 21.1$ & $114.9 \pm 14.5^{* *}$ & $96.6 \pm 26.9$ & $97.3 \pm 29.3$ \\
\hline Flexion, $\% B w$ & $61.8 \pm 19.1$ & $73.8 \pm 18.5^{* *}$ & $62.5 \pm 16.9$ & $67.2 \pm 24.4$ \\
\hline \multicolumn{5}{|l|}{ Flexibility } \\
\hline Anteflexion of the trunk $(\mathrm{cm})$ & $-0.9 \pm 12.2$ & $3.1 \pm 9.7 * *$ & $-1.6 \pm 9.8$ & $-1.4 \pm 9.4$ \\
\hline \multicolumn{5}{|l|}{ Daily phisical activity } \\
\hline Mean step counts (step/day) & $7,887 \pm 2,889$ & $8,069 \pm 3,149$ & $6,428 \pm 2,366$ & $6,385 \pm 2,752$ \\
\hline Calculated mean energy ( $k$ cal/day) & $220 \pm 101$ & $225 \pm 100$ & $187 \pm 93$ & $184 \pm 104$ \\
\hline
\end{tabular}

Values are mean $\pm S D . * p<0.05$ compared with baseline, ${ }^{*} p<0.01$ compared with baseline.

Peak $\dot{V} O 2$, peak oxygen consumption; AT, anaerobic threshold. Other abbreviation see in Table 1.

Table 3 Effects of Cardiac Rehabilitation and Exercise Training on Lipid Profile and Other Parameters

\begin{tabular}{lccccc}
\hline \hline & \multicolumn{2}{c}{ Intervention group } & & \multicolumn{2}{c}{ Control group } \\
\cline { 2 - 3 } \cline { 5 - 6 } & At baseline & After 6 months & & At baseline & After 6 months \\
\hline$T C(\mathrm{mg} / \mathrm{dl})$ & $188 \pm 27$ & $179 \pm 28^{*}$ & & $193 \pm 15$ & $188 \pm 18$ \\
$T G(\mathrm{mg} / \mathrm{dl})$ & $115 \pm 46$ & $121 \pm 59$ & & $132 \pm 80$ & $130 \pm 53$ \\
$H D L-C(\mathrm{mg} / \mathrm{dl})$ & $50 \pm 13$ & $47 \pm 10^{*}$ & & $53 \pm 13$ & $52 \pm 12$ \\
LDL $-\mathrm{C}(\mathrm{mg} / \mathrm{dl})$ & $115 \pm 20$ & $107 \pm 22$ & & $113 \pm 20$ & $110 \pm 22$ \\
ApoA- $(\mathrm{mg} / \mathrm{dl})$ & $130 \pm 22$ & $123 \pm 21^{* *}$ & & $136 \pm 28$ & $135 \pm 23$ \\
ApoB $(\mathrm{mg} / \mathrm{dl})$ & $104 \pm 16$ & $102 \pm 19$ & & $108 \pm 16$ & $106 \pm 20$ \\
FBS $(\mathrm{mg} / \mathrm{dl})$ & $99 \pm 19$ & $95 \pm 15$ & & $103 \pm 23$ & $97 \pm 20$ \\
Hemoglobin Alc $(\%)$ & $5.7 \pm 0.7$ & $5.8 \pm 0.7$ & & $6.1 \pm 1.2$ & $6.0 \pm 1.0$ \\
\cline { 5 - 6 } & & &
\end{tabular}

Values are mean $\pm S D . * p<0.05$ compared with baseline, $* * p<0.01$ compared with baseline.

$T C$, total cholesterol; TG, triglycerides; HDL-C, high-density lipoprotein-cholesterol; LDL-C, low-density lipoprotein-cholesterol; Apo, apolipoprotein; FBS, fasting blood sugar.

\section{Results}

\section{Baseline Characteristics}

The baseline characteristics of the intervention and control groups are presented in Table 1 . The mean age of both groups was relatively high because of the inclusion criteria. Age and BMI, as well as the prevalence of smoking, hypertension, and diabetes and other clinical characteristics, were not statistically different between groups.

\section{Parameters After 6 Months of Cardiac Rehabilitation}

No subject in either group showed any worsening of symptoms or had clinical events during this study.

Obesity Indices Obesity indices at baseline and after 6 months of rehabilitation in each group is shown in Table 2 . Obesity indices and daily physical activity were not significantly different at baseline between the 2 groups. In the intervention group, the BMI (from 23.4 \pm 2.6 to $22.9 \pm$ $2.5 \mathrm{~kg} / \mathrm{m}^{2}, \mathrm{p}<0.01$ ), waist circumference (from $86.1 \pm 7.3$ to $83.9 \pm 7.3 \mathrm{~cm}, \mathrm{p}<0.01$ ), and fat weight (from $16.2 \pm 4.3$ to $15.2 \pm 3.6 \mathrm{~kg}, \mathrm{p}<0.05)$ significantly decreased and the lean body weight was maintained (from $45.8 \pm 4.8$ to $45.5 \pm 5.1 \mathrm{~kg}$, NS). In the control group, none of the parameters changed.

Physical Activity and Exercise Tolerance Exercise tolerance, the strength of the thigh muscles, flexibility of the trunk, and daily physical activity at baseline and after 6 months of rehabilitation are presented in Table 2. Based on the records of the Lifecorder, daily physical activity did not remarkably improve in either group. The mean step count of the intervention group and of the control group after 6 months went from $7,887 \pm 2,889$ at baseline to $8,069 \pm 3,149$ steps/day, and from $6,428 \pm 2,366$ to $6,385 \pm 2,752$ steps/day, respectively. The calculated mean energy expenditure of physical activity in the intervention group and the control group went from $220 \pm 101$ to $225 \pm 109 \mathrm{kcal} / \mathrm{day}$, and $187 \pm$ 93 to $184 \pm 104 \mathrm{kcal} / \mathrm{day}$, respectively. In the intervention group, peak $\dot{\mathrm{V}}_{2}$ and $\mathrm{AT} \dot{\mathrm{V}} \mathrm{O}_{2}$ tended to increase after 6 months of rehabilitation (from $22.8 \pm 3.4$ to $24.6 \pm 3.1 \mathrm{ml}$. $\mathrm{kg}^{-1} \cdot \mathrm{min}^{-1}$ and $12.4 \pm 1.6$ to $13.2 \pm 2.0 \mathrm{ml} \cdot \mathrm{kg}^{-1} \cdot \mathrm{min}^{-1}$, respectively). In the control group, however, peak $\mathrm{V}_{2}$ significantly decreased (from $24.9 \pm 3.7$ to $22.4 \pm 3.2 \mathrm{ml} \cdot \mathrm{kg}^{-1} \cdot \mathrm{min}^{-1}$, $\mathrm{p}<0.01$ ) and the $\mathrm{AT} \mathrm{V}_{2}$ did not change during the study period (from $12.6 \pm 1.8$ to $12.0 \pm 2.0 \mathrm{ml} \cdot \mathrm{kg}^{-1} \cdot \mathrm{min}^{-1}$ ). In the intervention group, the isokinetic peak torques of the knee extensor and flexor muscles were significantly increased after 6 months of rehabilitation. In the control group, none of the parameters changed. Moreover, flexibility defined by anteflexion of the trunk was significantly improved in the intervention group (from $-0.9 \pm 12.2$ to $3.1 \pm 9.7 \mathrm{~cm}$ ), but not in the control group (from $-1.6 \pm 9.8$ to $-1.4 \pm 9.4 \mathrm{~cm}$ ). 
Serum Lipid Profiles and Glucose Parameters Serum lipid profiles at baseline and after 6 months of rehabilitation are presented in Table 3. Lipid profiles at baseline were not significantly different between the 2 groups. In the intervention group, the serum TC concentration was significantly decreased after 6 months rehabilitation when compared with baseline (from $188.1 \pm 26.9$ to $178.7 \pm 28.3 \mathrm{mg} / \mathrm{dl}$, $\mathrm{p}<0.05)$. The serum concentrations of HDL-C and apoA-I also significantly decreased, but TG did not change during the study period. In the control group, serum lipid profiles did not change at all. In the intervention group, levels of fasting blood sugar tended to decrease (from $98.6 \pm 18.9$ to $94.9 \pm 14.5 \mathrm{mg} / \mathrm{dl}, \mathrm{p}=0.055$ ), although hemoglobin A1c remained unchanged. In the control group, none of the parameters changed.

\section{Discussion}

Most studies of cardiac rehabilitation in Japan have examined the effectiveness of phase I or II programs and there is very limited data available regarding phase III. Patients with CAD in the chronic phase still have problems with physical function and coronary risk factors, especially if they are elderly!2,13,15-17 The J-CARP was designed to examine the effects of an outpatient phase III cardiac rehabilitation program on these parameters in patients over 65 years old with CAD enrolled in a prospective randomized controlled trial ${ }^{1}$ In the present study, elderly CAD patients in the intervention group showed reduced BMI and fat weight without reduction of lean body weight. Although the intervention group was unable to demonstrate a significant improvement of physical fitness, they maintained exercise tolerance for 6 months in contrast to the control group, in which the peak $\dot{\mathrm{V}}_{2}$ significantly decreased. In addition, the intervention group showed significantly improved lower muscle strength. Lipid profiles did not show any significant improvement, except for decreased of TC, which may be countered by the decrease in HDL-C levels. These results suggest that cardiac rehabilitation in elderly CAD patients may have beneficial effects on physical function, but may not be sufficient to improve coronary risk factors such as the lipid profile.

\section{Physical Fitness}

In general, peak $\dot{\mathrm{V}}_{2}$ is progressively reduced by the aging process itself or in combination with various diseases in elderly subjects 17,18 Previous studies of elderly participants have indicated a diminished exercise capacity compared with younger subjects; however, similar improvements in aerobic capacity have been observed after exercise training! 19 Ades and Grunvald demonstrated that elderly patients were usually substantially less fit; however, they obtained greater improvements in their aerobic capacity as shown by enhanced peak oxygen consumption and duration of treadmill exercise after cardiac rehabilitation ${ }^{20}$ On the other hand, Lavie et al reported that improvements in cardiopulmonary variables, such as peak $\dot{\mathrm{V}} \mathrm{O}_{2}$, were relatively small in elderly participants? Compared with several previous studies performed in other countries, ${ }^{79-21}$ in the present study oxygen consumption was already at higher levels at baseline, which may be why no significant improvement in peak $\dot{\mathrm{V}}_{2}$ was observed in the intervention group. Improvements in aerobic exercise capacity are greater in patients with lower exercise capacities than in those with higher exercise tolerance? Elderly patients with CAD have high rates of disability, partly because of the weakness of the skeletal muscles 19 Ades et al reported that even activities considered aerobic, such as walking, were limited by reduced leg muscle strength in elderly patients?2 In elderly patients, improvement of thigh muscle strength may aid in the maintenance of an active lifestyle. A report from the WHO also suggests that improved oxygen efficiency in peripheral skeletal muscle and increased muscle strength may benefit the long-term prognosis? 23 Therefore, we believe that comprehensive cardiac rehabilitation is still an important strategy, even if it cannot improve exercise tolerance in elderly patients with CAD.

\section{Obesity Indices and Lipid Profiles}

In the intervention group, a significant reduction of BMI and percent body fat was observed without a loss of lean body weight after 6 months of cardiac rehabilitation. However, the concentrations of not only TC, but also HDL-C and apoA-I were significantly decreased. It has been frequently reported that cardiac rehabilitation brings favorable changes in plasma lipoprotein levels, such as reductions of $\mathrm{TC}$ and TG, as well as a significant increase in the HDL-C level? ? $^{2}$ The present program consisting of a weekly supervised exercise session at the clinic with 2 home-based exercise sessions may not be sufficient to increase HDL-C or apoA-I levels. In addition, the subjects in the intervention group were under strict diet therapy during this program. Therefore, HDL-C and apoA-I may have decreased as described previously? ${ }^{4}$

\section{Study Limitations}

There are several potential limitations to the present study. First, some of the negative results may be a result of the small sample size. Second, as discussed earlier, the supervised exercise session at the outpatient clinic was performed only once a week with at least 2 exercise sessions at home. These parameters might not be enough to increase the exercise capacity and to change the metabolic status when compared with previous programs. Third, the present study consisted only of male subjects who were selected from an outpatient clinic. Therefore, the results of this study may not be representative of all elderly patients with CAD.

\section{Conclusion}

Phase III comprehensive cardiac rehabilitation in the intervention group partially but significantly improved several aspects of physical fitness and coronary risk, suggesting that cardiac rehabilitation in the chronic phase may benefit elderly patients with CAD. Further studies with large sample sizes are required to confirm the efficacy of cardiac rehabilitation in the elderly population.

\section{Acknowledgments}

This study was supported by Health Sciences Research Grants from Ministry of Health, Labour and Welfare (Comprehensive Research on Aging and Health). The authors wish to thank each of the participants for their data.

\section{References}

1. Lavie CJ, Milani RV. Factors predicting improvements in lipid values following cardiac rehabilitation and exercise training. Arch Intern Med 1993; 153: 982-988.

2. Lavie CJ, Milani RV, Littman AB. Benefits of cardiac rehabilitation and exercise training in secondary coronary prevention in the elderly. J Am Coll Cardiol 1993; 22: 678-683. 
3. Lavie CJ, Milani RV. Effects of cardiac rehabilitation programs on exercise capacity, coronary risk factors, behavioral characteristics, and quality of life in a large elderly cohort. Am J Cardiol 1995; 76: $177-179$.

4. Lavie CJ, Milani RV. Effects of cardiac rehabilitation and exercise training programs in patients $>$ or $=75$ years of age. Am J Cardiol 1996; 78: $675-677$.

5. Milani RV, Lavie CJ. Behavioral differences and effects of cardiac rehabilitation in diabetic patients following cardiac events. Am J Med 1996; 100: $517-523$

6. Milani RV, Lavie CJ. Prevalence and effects of cardiac rehabilitation on depression in the elderly with coronary heart disease. Am J Cardiol 1998; 81: $1233-1236$.

7. Lavie CJ, Milani RV. Disparate effects of improving aerobic exercise capacity and quality of life after cardiac rehabilitation in young and elderly coronary patients. J Cardiopulm Rehabil 2000; 20: 235-240.

8. O'Connor GT, Buring JE, Yusuf S, Goldhaber SZ, Olmstead EM, Paffenbarger RS Jr, et al. An overview of randomized trials of rehabilitation with exercise after myocardial infarction. Circulation 1989; 80: $234-244$

9. Ades PA, Waldmann ML, McCann WJ, Weaver SO. Predictors of cardiac rehabilitation participation in older coronary patients. Arch Intern Med 1992; 152: 1033-1035.

10. Pasquali SK, Alexander KP, Peterson ED. Cardiac rehabilitation in the elderly. Am Heart J 2001; 142: 748-755.

11. Seki E, Watanabe Y, Sunayama S, Iwama Y, Shimada K, Kawakami $\mathrm{K}$, et al. Effects of phase III cardiac rehabilitation programs on healthrelated quality of life in elderly patients with coronary artery disease. Circ J 2003; 67: 73-77.

12. Goto $\mathrm{Y}$, Itoh H, Adachi H, Ueshima K, Nohara R. Use of exercise cardiac rehabilitation after acute myocardial infarction. Circ J 2003; 67: $411-415$

13. Goto Y, Saito M, Iwasaka T, Daida H, Kohzuki M, Ueshima K, et al; The Japanese Cardiac Rehabilitation Survey Investigators. Poor implementation of cardiac rehabilitation despite broad dissemination of coronary interventions for acute myocardial infarction in Japan. Circ J 2007; 71: 173-179.

14. Gibbons RJ, Balady GJ, Bricker JT, Chaitman BR, Fletcher GF, Froelicher VF, et al, American College of Cardiology/American
Heart Association Task Force on Practice Guidelines (Committee to Update the 1997 Exercise Testing Guidelines). ACC/AHA 2002 guideline update for exercise testing: Summary article: A report of the American College of Cardiology/American Heart Association Task Force on Practice Guidelines (Committee to Update the 1997 Exercise Testing Guidelines). Circulation 2002; 106: 1883-1892.

15. Hellman EA, Williams MA. Outpatient cardiac rehabilitation in elderly patients. Heart Lung 1994; 23: 506-512.

16. Yoshida T, Yoshida K, Yamamoto C, Nagasaka M, Tadaura H, Meguro T. Effects of a two-week, hospitalized phase II cardiac rehabilitation program on physical capacity, lipid profiles and psychological variables in patients with acute myocardial infarction. Jpn Circ J 2001; 65: 87-93.

17. Ades PA, Waldmann ML, Meyer WL, Brown KA, Poehlman ET, Pendlebury WW, et al. Skeletal muscle and cardiovascular adaptations to exercise conditioning in older coronary patients. Circulation 1996; 94: 323-330.

18. Fleg JL, Morrell CH, Bos AG, Brant LJ, Talbot LA, Wright JG, et al. Accelerated longitudinal decline of aerobic capacity in healthy older adults. Circulation 2005; 112: 674-682.

19. Ades PA, Waldmann ML, Gillespie C. A controlled trial of exercise training in older coronary patients. J Gerontol A Biol Sci Med Sci 1995; 50A: M7-M11.

20. Ades PA, Grunvald MH. Cardiopulmonary exercise testing before and after conditioning in older coronary patients. Am Heart J 1990; 120: $585-589$.

21. Ades PA, Savage PD, Brawner CA, Lyon CE, Ehrman JK, Bunn JY, et al. Aerobic capacity in patients entering cardiac rehabilitation. Circulation 2006; 113: 2706-2712.

22. Ades PA, Ballor DL, Ashikaga T, Utton JL, Nair KS. Weight training improves walking endurance in the healthy elderly persons. Ann Intern Med 1996; 124: 568-572.

23. WHO Expert Committee. Physical status: The use and interpretation of anthropometry. WHO Tech Report Ser 1995; 854: 375-409.

24. Fiatarone MA, O'Neill EF, Ryan ND, Clements KM, Solares GR, Nelson ME, et al. Exercise training and nutritional supplementation for physical frailty in very elderly people. $N$ Engl J Med 1994; 330: $1769-1775$. 